\title{
Propuesta metodológica para la integración del sistema de gestión de la calidad basado en la NTC ISO 9001:2008 y los procesos de acreditación de alta calidad del CNA*
}

Methodological proposal for the integration of the quality management system based on the NTC ISO 9001: 2008 and the processes of accreditation of high quality of the CNA

Recibido: 6 de abril de 2016 Revisado: 25 de abril de 2016 Aceptado: 13 de junio de 2016

Myriam Góme: Colmenares"

Universidad Santo Tomás

Ximena Lucía Pedraza Najar "**

Convenio USTA - Icontec

Artículo de resultado de investigación.

** Economista. Especialista en Finanzas. Especialista en Gerencia de Instituciones de Educación Superior. Magíster (c) en Calidad y Gestión Integral. Secretaria académica en la Universidad Santo Tomás. Correo electrónico: myriamgomez@usantotomas.edu.co

*** Microbióloga industrial. Especialista en Gestión de la Producción, la Calidad y la Tecnología. Especialista en Gerencia de Procesos, Calidad e Innovación. Magíster en Calidad y Gestión Integral. Doctorado en curso en Administración. Correo electrónico: ximena.pedraza@hotmail.com 


\section{RESUMEN}

Este artículo es el resultado de la reflexión metodológica sobre la relación que podría establecerse entre el sistema de gestión de la calidad, según la norma técnica ISO 9001:2008 y el proceso de acreditación de alta calidad para programas de pregrado de la educación superior del Consejo Nacional de Acreditación de Colombia. Propone una metodología que considera aspectos y requisitos de los referentes mencionados, para construir una herramienta de carácter consultivo y establecer el apoyo de un referente sobre el otro. Los referentes analizados están vinculados a la Universidad Santo Tomás, en donde se observa que el desempeño de la organización es afectado por la duplicidad de actividades y procesos relativos al aseguramiento de la calidad.

Palabras clave: educación superior, sistema de gestión de la calidad, acreditación, ISO 9001.

\section{ABSTRACT}

This article is the result of methodological reflection on the relationship that could be established between the quality assurance system, according to ISO 9001: 2008 and the high quality accreditation process for undergraduate higher education programs of the Consejo Nacional de Acreditación in Colombia. It proposes a methodology that considers aspects and requirements of the referents mentioned, to build a tool of an advisory nature and establish the support of one referent over the other. The referents analyzed are linked to the Universidad Santo Tomás, where it is observed that the performance of the organization is affected by the duplication of activities and processes related to quality assurance.

Keywords: Higher education, quality management systems, quality assurance, ISO standards.

\section{INTRODUCCIÓN}

Las instituciones de educación superior en Colombia realizan diversas acciones tendientes a promover, integrar, evaluar y gestionar la mejora continua de los procesos académicos y administrativos en las facultades y programas, con el objeto de lograr una educación de alta calidad.

El informe de aseguramiento de la calidad en Iberoamérica, desarrollado en el 2012 por el Centro Interuniversitario de Desarrollo (CINDA), reafirma lo que seguramente muchos académicos en la cotidianidad de la gestión han percibido: las universidades son las responsables de asegurar la calidad integral y el desarrollo armónico de las actividades formadoras y de gestión, a través de mecanismos adecuados para el mejoramiento de la calidad, de tal manera que se constituyan en incentivos para aplicar procesos de gestión de calidad y de autorregulación permanentes (Lemaitre y Zenteno 2012).

En Colombia, las universidades se rigen por el sistema de aseguramiento de la calidad establecido por el Ministerio de Educación Nacional (MEN), a través del Consejo Nacional de Acreditación (CNA), según la Constitución Política de Colombia de 1991 y las leyes y decretos correspondientes. Existen también los lineamientos establecidos por el Sistema Nacional de Acreditación (SNA), de acuerdo con el artículo 53 de la Ley 30 de 1992 que complementan dicha normatividad.

La acreditación de los programas de pregrado en la educación superior la otorga el MEN, siguiendo los procesos de autorregulación y autoevaluación al interior de las Instituciones de Educación Superior (IES) y la evaluación externa realizada por el CNA, organismo encargado de verificar el cumplimiento de las condiciones de calidad, que le permiten pronunciarse y sugerir acciones de mejora. 
El sistema de gestión de la calidad (SGC), según los requisitos establecidos por la norma ISO 9001:2008, hace parte de los procesos de planeación y orienta a los programas con el propósito de llevar a cabo los controles, verificaciones de las condiciones que contribuyen a la mejora de procesos, los criterios para la conservación de evidencias necesarias para que se logren y cumplan los estándares de calidad. Las políticas, directrices y líneas para el desarrollo de los procesos las determina la directiva institucional.

El SGC lo asume la Universidad, como un medio para la construcción de una cultura de mejoramiento continuo que mediante la recolección de evidencias, las verificaciones, articulación y seguimiento de los procesos apoya las distintas dinámicas de gestión para el logro de los objetivos propios de las funciones sustantivas de docencia, investigación y proyección social, propios de la educación superior.

En la Universidad Santo Tomás (USTA) coexisten los dos procesos para el aseguramiento de la calidad: acreditación de alta calidad de programas de pregrado y el sistema de gestión de la calidad orientado con los lineamientos de la NTC ISO 9001:2008, que se ejecutan con metodologías y funciones distintas.

Las estrategias y procesos para la acreditación de alta calidad de los programas de pregrado se adelantan según los lineamientos y parámetros del CNA y otras disposiciones del MEN, orientadas hacia el cumplimiento de los objetivos institucionales, los objetivos del registro calificado, de acreditación y reacreditación de alta calidad de los programas académicos.

Sin embargo, la falta de integración entre los dos referentes mencionados afecta el desempeño organizacional, en la medida en que se emplee más tiempo repitiendo actividades, de manera independiente, sin concretar una solución en donde se involucre un todo. Es necesario encontrar una forma de integrar los procesos y aplicarlos de manera que uno apoye a otro y se superen las duplicidades de actividades e información.

En este artículo se propone una metodología para desarrollar una herramienta que facilite la toma de decisiones de la institución en orden de alcanzar el aseguramiento de la calidad y contribuya a que la información concerniente al CNA pueda relacionarse de manera efectiva con los aspectos de la norma ISO 9001:2008

\section{METODOLOGÍA}

La metodología propuesta para la integración de un sistema de gestión de la calidad basado en la norma NTG ISO 9001:2008 y los procesos de acreditación de alta calidad del CNA, proviene de la necesidad de identificar los aspectos, factores y características en que interactúan los dos referentes. La metodología propone cinco momentos: 1) diagnóstico; 2) evaluación de la articulación; 3) formulación del plan de intervención; 4) ejecución del plan de intervención; 5) evaluación del plan de intervención (figura 1).

El momento de diagnóstico busca conocer las condiciones, requisitos y aspectos entre los que coexisten los dos referentes, este momento constituye el insumo principal del presente trabajo. Los demás momentos no se desarrollan en este artículo, pero se proponen como pasos posteriores a la formulación del diagnóstico, el cual es el punto de partida para la aplicación de la metodología propuesta. 
Figura 1. Metodología propuesta para determinar la articulación entre el sistema de gestión de la calidad y acreditación de programas de pregrado (SGC - APP).

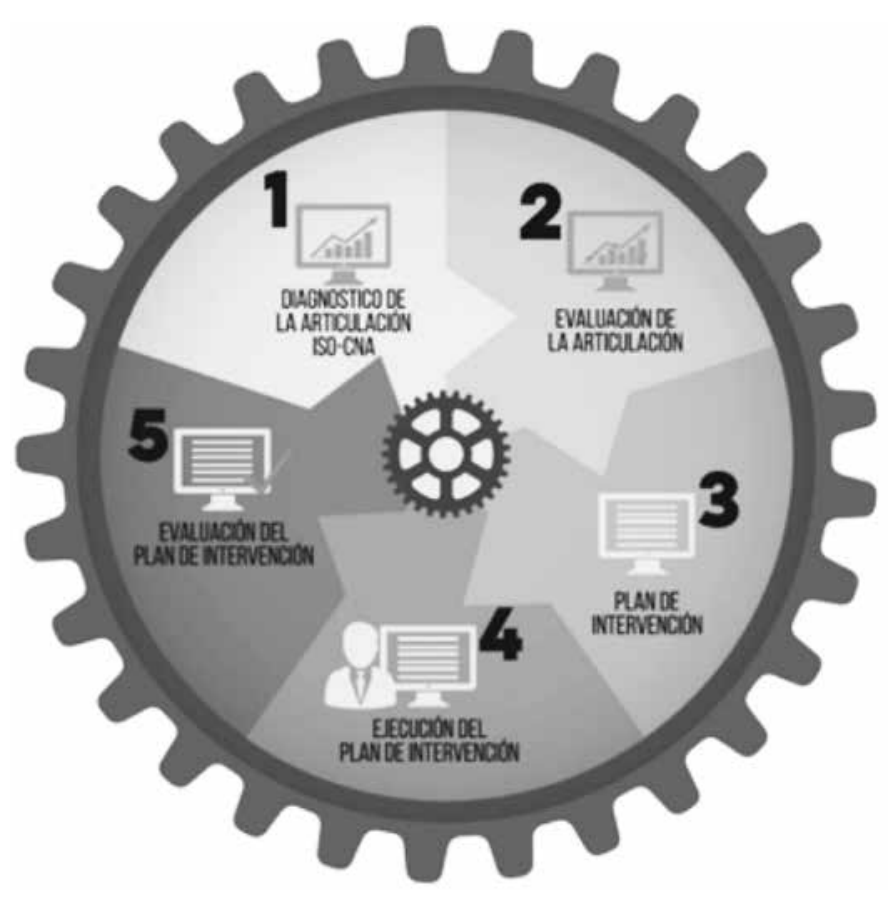

Fuente: elaboración propia.

\section{Momento uno, el diaǵnóstico}

Las actividades desarrolladas en el momento del diagnóstico se muestran en la Figura 2.

La actividad uno lleva a obtener y disponer de cada uno de los 10 factores con sus 40 características y 253 aspectos a evaluar, correspondientes al proceso de acreditación de alta calidad para programas de pregrado del CNA; igualmente se identifican 8 requisitos y cerca de 150 procesos y actividades articulados a partir de lo determinado por el enfoque basado en procesos según la norma ISO 9001:2008.

Identificados los procesos, actividades y requisitos, se procede a determinar los requisitos del SGC que interactúan con cada uno de los procesos y características del sistema de acreditación CNA y viceversa. En la tabla 1 se listan en términos generales los capítulos desarrollados por la norma ISO 9001:2008, en correspondencia con los lineamientos para la acreditación de programas de pregrado del CNA.

Fiǵura 2. Actividades del momento del diagnóstico.

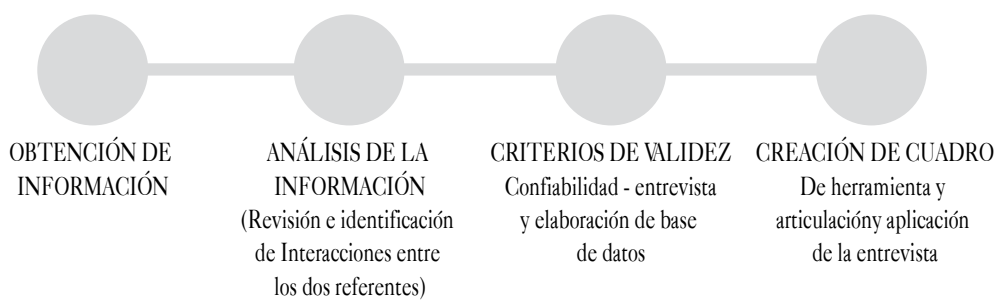

Fuente: elaboración propia. 
Propuesta metodológica para la integración del sistema de gestión de la calidad basado en la NTC ISO 9001:2008 y los procesos de acreditación de alta calidad del CNA

Tabla 1. Capítulos de la norma ISO 9001:2008 y lineamientos de los referentes analizados del CNA.

\begin{tabular}{|l|l|}
\hline \multicolumn{1}{|c|}{ CAPÍTULOS NORMA ISO 9001:2008 } & \multicolumn{1}{c|}{ LINEAMIENTOS DE CNA } \\
\hline 4. Sistema de gestión de la calidad & 1. Misión, Proyecto Institucional y de Programa \\
\hline 5. Responsabilidad de la dirección & 2. Estudiantes \\
\hline 6. Gestión de los recursos & 3. Profesores \\
\hline 7. Realización del producto & 4. Procesos Académicos \\
\hline 8. Medición, análisis y mejora & 5. Visibilidad Nacional e Internacional \\
\hline & 6. Investigación, Innovación y Creación \\
\hline & 7. Bienestar Institucional \\
\hline & 8. Organización, Administración y Gestión \\
\hline & 9. Impacto de los Egresados en el Medio \\
\hline & 10. Recursos Físicos y Financieros \\
\hline
\end{tabular}

Fuente: autoría propia.

De esta manera se desarrollan hasta aquí dos actividades, en este momento, de diagnóstico: 1) la recolección de información; 2) el estudio y análisis de los factores del APP y procesos y actividades del SGC.

La actividad tres se fundamenta en la revisión de la información aportada por el contexto general y la obtenida de la realidad de la USTA, en cuyo interior se dan las interacciones propias de una comunidad académica y de los consecuentes procesos de aseguramiento de la calidad, con la intención de profundizar y aportar a la mejora de los procesos de articulación desarrollados en pos los dos referentes analizados. Para conocer la percepción de las partes interesadas y los aspectos en los que se evidencia una articulación e interacción real en la operación de los dos referentes, se utiliza como criterio de validez y confiabilidad una entrevista semiestructurada.

Se destacan algunas respuestas, que se relacionan por la frecuencia manifestada por las partes interesadas de la USTA, así:

En cuanto a la percepción de la articulación del SGC y los procesos para APP:
1. La articulación entre los dos procesos es incipiente.

2. Existe muy poca articulación de los procesos, debe avanzar y mostrar más resultados.

En relación con los procesos adelantados en las verificaciones y evaluaciones hacia el mejoramiento de la calidad se manifiestan las partes interesadas:

1. Los resultados de ambos procesos de calidad sí generan información útil a la calidad de los programas, aunque en mayor grado lo hace el proceso de autoevaluación (CNA).

2. Los resultados del proceso de autoevaluación-autorregulación presentan mayores aportes a la calidad de los programas académicos.

3. En el proceso de toma de decisiones los resultados de ambos procesos aportan información valiosa para el mejoramiento de calidad, tanto del programa, las facultades y la división, pero de manera aislada.

La actividad cuatro parte de la utilización de hojas de cálculo de Excel, a través de las cuales se desarrolla una base de datos, que por medio del uso de tablas 
dinámicas facilita la identificación de las relaciones de requisitos, factores y aspectos en común en los dos referentes analizados (figura 3 y figura 4).

A manera de ejemplo, el recuadro verde de la figura 3 , muestra el literal del sistema de gestión de la calidad 4.1.a, que una vez seleccionado, despliega los aspectos del proceso de acreditación del CNA, con los que dicho requisito se relaciona (1.1.c, 2.4.c., etc.), según lo desarrollado en las actividades 1 y 2. Cabe anotar que el literal 4.1.a del CGS, hace referencia al numeral 4: sistema de gestión de la calidad; al subnumeral 1: requisitos generales; $\mathrm{y}$ literal a: determinar los procesos necesarios para el sistema de gestión de la calidad y su aplicación a través de la organización.

Figura 3. Cuadro diagnóstico que muestra la relación ISO-CNA.

\begin{tabular}{|c|c|l|}
\hline \multicolumn{2}{|c|}{ LITERAL SISTEMA DE GESTIÓN DE LA CALIDAD } \\
\hline 4.1.a determinar los procesos necesarios para el sistema de gestión de la calidad y su aplicación a través de la organización \\
\hline \multicolumn{2}{|c|}{ RELACIÓN CON ASPECTO A EVALUAR CNA } \\
\hline 1 & 1.1.c & $\begin{array}{l}\text { El proyecto institucional orienta las acciones y decisiones de programa académico, en la gestión del currículo, la docencia, } \\
\text { la investigación científica, la creación artística, la internacionalización, la proyección social, el bienestar de la comunidad } \\
\text { institucional y demás áreas estratégicas de la institución }\end{array}$ \\
\hline 2 & $2.4 . c$ & $\begin{array}{l}\text { Existencia y utilización de sistemas y mecanismos de evaluación de los procesos de selección y admisión, y aplicación de } \\
\text { los resultados de dicha evaluación }\end{array}$ \\
\hline 4 & 2.4.d & $\begin{array}{l}\text { Requerimientos para el ingreso de estudiantes en condición de transferencia, homologación y otro proceso que amerite } \\
\text { criterios específicos para el tránsito entre ciclos, niveles y/o istituciones. Beneficios de estos requerimientos en formación } \\
\text { integral de }\end{array}$ \\
\hline 6 & $4.16 . a$ & $\begin{array}{l}\text { Existencia de criterios y mecanismos para el seguimiento y la evaluación del desarrollo de competencias, especialmente las } \\
\text { actitudes, los conocimientos, las capacidades y las habilidades generales y aquellas que son específicas del ejercicio y de la } \\
\text { cultura de la profesión o la disciplina en la que se forma al estudiante }\end{array}$ \\
\hline 4.19.k & $\begin{array}{l}\text { Estrategias para garantizar el éxito académico de los estudiantes en el tiempo para el desarrollo del plan de estudios, } \\
\text { atendiendo los estándares de calidad }\end{array}$ \\
\hline
\end{tabular}

Fuente: elaboración propia.

Figura 4. Cuadro Diagnóstico que muestra la relación CNA-ISO.

\begin{tabular}{|l|l|l|}
\hline \multicolumn{2}{|c|}{ ASPECTO A EVALUAR DEL CNA } \\
\hline $\begin{array}{l}\text { 1.1.c El proyecto institucional orienta las acciones y decisiones del programa académico, en la gestión del currículo, la docencia, la creación } \\
\text { artística, la internacionalización, la proyección social, el bienestar de la comunidad institucional y demás áreas estratégicas de la institución }\end{array}$ \\
\hline \multicolumn{2}{|c|}{ LITERALES DEL SGC QUE SE CORRELACIONAN CON EL ASPECTO A EVALUAR 1.1.c } \\
\hline 1 & 4.1 & SISTEMAS DE GESTIÓN DE LA CALIDAD Requisitos Generales \\
\hline 2 & $4.1 . a$ & determinar la secuencia e interacción de estos procesos \\
\hline 3 & 4.1.f & $\begin{array}{l}\text { La documentación del sistema de gestión de la calidad debe incluir: declaraciones documentadas de una política de la } \\
\text { calidad y de objetivos de la calidad }\end{array}$ \\
\hline 4 & 4.2.2.a & Control de los documentos \\
\hline
\end{tabular}

Fuente: elaboración propia. 
El uso de esta herramienta facilita la consulta de aquellos aspectos definidos para la acreditación de programas de pregrado, en los cuales hay interacción con los requisitos asociados al SGC, necesidad que continuamente se requiere dada la insistencia en el argumento de desconocimiento de la aplicabilidad de los requisitos del SGC, y mucho más de la articulación que entre los dos referentes pueda existir, la actividad tres del desarrollo del proceso de diagnóstico permitió conocer algunos argumentos de las partes interesadas en los procesos de aseguramiento de la calidad.

\section{RESULTADOS Y DISCUSIÓN}

Los resultados obtenidos del trabajo realizado a través de la metodología propuesta en lo concerniente a la fase diagnóstica se resumen en:

1. Se evidenció que hay elementos comunes en los dos procesos de calidad que tiene la USTA y que permiten consolidar los planes de mejoramiento.

2. Las opiniones encontradas en algunas respuestas de la entrevista también reflejan las contradicciones que se dan al interior de los dos sistemas y que dificultan la integración de los procesos.

3. Se coincide en que existen elementos, factores o lineamientos particulares a cada sistema, que deben manejarse de forma independiente, ya que no se puede realizar una articulación entre ellos.

4. El $38 \%$ de los entrevistados reconoce que el sistema de gestión de la calidad aporta a la organización de la gestión documental de los procesos de calidad en los programas académicos.

5. Los resultados de la aplicación de los procesos de los dos referentes generan información útil para asegurar la calidad de los programas, aunque esta utilidad se hace más evidente con el proceso de autoevaluación (CNA).

6. Un $50 \%$ de los entrevistados considera que los planes de mejoramiento, informes de auditoría, informes de gestión son, entre otros, evidencias generadas que aplican para ambos procesos de calidad.

La realidad observada y la experiencia de la USTA, permite ver el gran valor que tiene la coexistencia entre el SGC y los lineamientos, actividades y gestión para alcanzar la acreditación de alta calidad de los programas de pregrado. Una clara muestra de lo anterior son las evidencias expresadas en los planes de mejoramiento, informes de auditoría e informes de gestión.

Las evidencias mencionadas dan cumplimiento a los requisitos de los referentes analizados y se desarrollan en concordancia con las funciones sustantivas, no obstante se encontró que las partes interesadas que participaron en la entrevista consideran que es importante que la alta dirección asuma los procesos de aseguramiento de la calidad, en la planeación estratégica de la USTA, no solo con la intencionalidad en orden al cumplimiento y más bien hacia el compromiso que conlleva la calidad institucional.

El procedimiento planteado implica un aporte a los compromisos con la calidad de la USTA, debido a que a través de la herramienta desarrollada expone de manera sistematizada la articulación de las actividades implementadas en torno al aseguramiento de la calidad.

Un potencial argumento para dar continuidad a la totalidad del procedimiento es el reconocimiento de una actualización de la norma ISO 9001:2008 a la versión 2015, que vincula, según se ha sabido a la fecha, el enfoque basado en riesgos, lo cual constituye para la presente investigación un insumo de actualización. 


\section{CONCLUSIONES}

La complementariedad entre el SGG y los procesos de acreditación de programas de pregrado (APP), se reconoce en términos conceptuales, sin embargo, es muy débil su articulación, en orden a alcanzar la acreditación de los programas, mediante los lineamientos dispuestos por el CNA y las particularidades de cada programa para el logro del mismo fin.

En relación con la falta de integración observada entre los dos referentes objetos del presente análisis, se concluye que una optimización de los procesos a través del reconocimiento de los aportes de un referente sobre el otro, puede ser el punto de partida para el logro de la articulación y mejora del desempeño organizacional.

Las distintas políticas, estrategias y acciones encaminadas al mejoramiento de la calidad de los programas de educación superior y de sus instituciones, requieren encontrar puntos de convergencia y complementariedad entre los dos referentes, dado su distinto origen y diversa metodología, para evitar actividades repetitivas e innecesarias.

Las personas responsables del desarrollo de los procesos relacionados con la calidad, de los programas académicos y de la aplicación de los instrumentos respectivos a los dos referentes de la USTA, deben conocer suficientemente los lineamientos, procesos y dinámicas de cada uno de ellos, así como los puntos de posible articulación, en orden a su aplicación eficiente y óptima de los aspectos involucrados.
La metodología propuesta debe involucrar la adaptación a las actualizaciones que se den en el marco de los referentes analizados, las cuales están fuera del alcance del presente trabajo y así dar continuidad al proceso de integración.

\section{REFERENCIAS}

Consejo Nacional de Acreditación CNA. (2013) Lineamientos para la acreditación de programas de pregrado. Bogotá: CNA.

Instituto Colombiano de Normas Técnicas y Certificación (Icontec). (2008). Sistemas de gestión de la calidad: Sistema de Gestión de la Calidad Requisitos, NTC - ISO 9001. Bogotá: Icontec.

Instituto Colombiano de Normas Técnicas y Certificación (Icontec). (2014). Normas fundamentales sobre gestión de la calidad y documentos de orientación para su aplicación, NTC ISO 9000. Bogotá: Icontec.

Lemaitre, M. J., y Zenteno, M. E. (2012). Fortalecimiento de la Calidad en Iberoamérica. Educación Superior Informe 2012. Chile: Centro Interuniversitario de Desarrollo - CINDA. Recuperado de http://www.cinda.cl/wp-content/ uploads/2014/02/Aseguramiento-de-la-calidaden-Iberoam\%C3\%A9rica-2012.pdf 